\title{
Keep Your Laziness in Check
}

\author{
KENNETH FONER, University of Pennsylvania, USA \\ HENGCHU ZHANG, University of Pennsylvania, USA \\ LEONIDAS LAMPROPOULOS, University of Pennsylvania, USA
}

We introduce StrictCheck: a property-based random testing framework for observing, specifying, and testing the strictness of Haskell functions. Strictness is traditionally considered a non-functional property; StrictCheck allows it to be tested as if it were one, by reifying demands on data structures so they can be manipulated and examined within Haskell.

Testing strictness requires us to 1) precisely specify the strictness of functions, 2) efficiently observe the evaluation of data structures, and 3) correctly generate functions with random strictness. We tackle all three of these challenges, designing an efficient generic framework for precise dynamic strictness testing. StrictCheck can specify and test the strictness of any Haskell function-including higher-order ones-with only a constant factor of overhead, and requires no boilerplate for testing functions on Haskell-standard algebraic data types We provide an expressive but low-level specification language as a foundation upon which to build future higher-level abstractions.

We demonstrate a non-trivial application of our library, developing a correct specification of a data structure whose properties intrinsically rely on subtle use of laziness: Okasaki's constant-time purely functional queue.

CCS Concepts: • Software and its engineering $\rightarrow$ Software testing and debugging; Software maintenance tools;

Additional Key Words and Phrases: Random Testing, Laziness, Haskell, Generic Programming

ACM Reference Format:

Kenneth Foner, Hengchu Zhang, and Leonidas Lampropoulos. 2018. Keep Your Laziness in Check. Proc. ACM Program. Lang. 2, ICFP, Article 102 (September 2018), 30 pages. https://doi.org/10.1145/3236797

\section{INTRODUCTION}

Lazy evaluation gives great power to functional programmers, enabling them to program with infinite data structures [Abel et al. 2013], transparently and efficiently memoize computations [Hinze 2000], and decompose programs into modular pipelines [Hughes 1989]. However, programming with laziness can come with its own unique frustrations. Incorrect use of laziness can result in subtle bugs: if a program is too lazy, it may suffer from memory leaks; if it is too strict, it may fall victim to asymptotic performance degradations and even infinite loops.

In practice, such bugs are often quite difficult to detect and diagnose. Seemingly trivial changes to one function can break the strictness of another function far away in a codebase. Moreover, a program with undesired strictness properties is often very similar to a program with the correct ones. They may differ from the desired implementation only when tested on infinite or diverging input data, or may be semantically equivalent but inferior in performance. Unfortunately, neither

Authors' addresses: Kenneth Foner, University of Pennsylvania, USA, kfoner@seas.upenn.edu; Hengchu Zhang, University of Pennsylvania, USA, hengchu@seas.upenn.edu; Leonidas Lampropoulos, University of Pennsylvania, USA, llamp@seas. upenn.edu.

This work is licensed under a Creative Commons Attribution 4.0 International License.

(C) 2018 Copyright held by the owner/author(s).

2475-1421/2018/9-ART102

https://doi.org/10.1145/3236797

Proc. ACM Program. Lang., Vol. 2, No. ICFP, Article 102. Publication date: September 2018. 
semantic divergence nor poor performance are necessary results of a strictness bug-so neither conventional property-based testing nor benchmarking are sufficient to fully diagnose these issues.

How, then, can we test and prevent these bugs during development? In this paper, we present StrictCheck: a property-based random testing framework extending QuickCheck [Claessen and Hughes 2000] to catch arbitrarily complex strictness bugs in Haskell programs.

StrictCheck allows the programmer to

- Specify strictness precisely, describing exactly what portion of its inputs a function is meant to evaluate,

- Observe the strictness of a function and reify this into a Haskell data structure using only a constant factor of overhead, and

- Test whether a function matches its strictness specification, reporting a minimal reproducible counterexample if it does not.

StrictCheck can test any function-including higher-order ones-defined over any data type, requiring no boilerplate for any Haskell 2010 algebraic data type. Moreover, StrictCheck is general enough to test functions over abstract types, existential types, and GADTs, given the appropriate typeclass instances.

\subsection{Strictly Defining Our Terms}

In a lazy language like Haskell, a value-yielding computation-a thunk-is executed only when that value is itself needed for some further computation. Consider the following program, where $f$ and $\mathrm{g}$ are some existing functions:

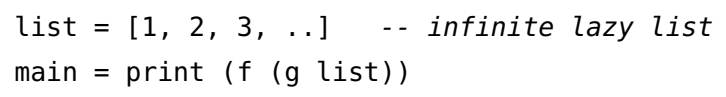

The program above prints all of f's result. To produce all of f's result, we need to evaluate some portion of g's result. In turn, to produce that portion of g's result, we need to evaluate some portion of list. Lazy evaluation proceeds by tracing the transitive dependencies of a series of such nested evaluation contexts.

A demand is the portion of a value required by some context. One possible demand on the list $[1,2,3, \ldots]$ above is $\left({ }_{-}: 2: \ldots\right)$. This notation says that some context evaluated the first two (:) constructors, as well as the second element of the list (the integer 2). The underscores indicate that neither the first element of the list nor the tail of the list were evaluated by the context. For our purposes, a demand represents a portion of a particular value, describing what actually happened to that value in one specific evaluation context. At a high level, you could view a demand as a pattern to match against: the pattern $\left({ }_{-}: 2:{ }_{-}\right)$evaluates the first two cons cells of a list, its second element, and nothing else.

The strictness of a function is a description of the demand that function exerts on its inputs, given a particular demand on its output and the particular values of its inputs. Strictness is not a mere boolean-evaluated or not-but a large spectrum of possible behaviors. For instance, some function

$$
f:: \text { Bool } \rightarrow \text { Bool } \rightarrow()
$$

could have one of nine different strictness behaviors, given a non-trivial demand on its result. Some of these include: evaluating neither argument; evaluating only the second argument; evaluating the first argument and evaluating the second if the first was was True; etc. Notice that any implementation of $f$ must be functionally equivalent to the constant function $\backslash x y \rightarrow($ ), modulo its behavior on undefined inputs. That is, two functions which are equivalent for all total inputs may have distinguishable strictness. 


\subsection{Describing Strictness, and Testing It!}

A strictness specification is a precise characterization of a function's strictness. Such a specification is a function itself, taking as input a particular demand on some function's output as well as a list of the function's inputs, and returning a list of predicted demands on those inputs. For example, if we have some function

$$
\mathrm{f}:: \mathrm{a} \rightarrow \mathrm{b} \rightarrow \ldots \rightarrow \mathrm{z} \rightarrow \text { result }
$$

then we might specify its demand behavior using another function

$$
\begin{aligned}
& \text { spec_f : : Demand result -- demand on function result } \\
& \rightarrow(a, b, \ldots, z) \quad \text { - - input values to function } \\
& \rightarrow \text { (Demand } a \text {, Demand } b, \ldots \text {, Demand } z \text { ) } \quad \text {-- demands on inputs }
\end{aligned}
$$

The structure of a specification suggests a natural flow for our testing framework, whose architecture is shown below in Figure 1.

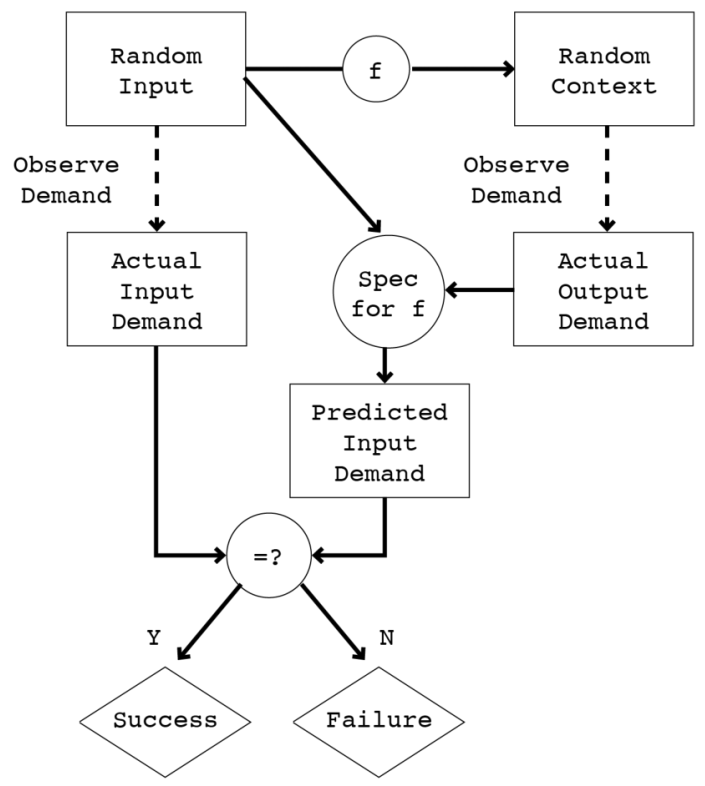

Fig. 1. Architecture of StrictCheck

In order to check whether a specification spec_f correctly predicts the demands on the inputs of a function $f$, we will

- generate random input(s) to $f:(a, b, \ldots, z)$,

- generate a random evaluation context $c$ for the result of $f$,

- evaluate $f$ in the context $c$ by forcing evaluation of ( $c$ ( $f \quad a \quad b \quad \ldots z)$ ),

- observe the demand $d$ which the context $c$ exerted on the result of $f$,

- observe the demands $(d a, d b, \ldots, d z)$ induced upon the inputs $(a, b, \ldots, z)$, and

- compare the actual observed demands $(d a, d b, \ldots, d z)$ with the predictions of the specification $(p d a, p d b, \ldots, p d z)=\operatorname{spec}_{-} f d a b \ldots z$,

repeating this until we've convinced ourselves that the specification holds.

In the next section we show how StrictCheck concretely represents specifications, using as an example the function take : : Int $\rightarrow[$ a $] \rightarrow[a]$. 
In the rest of the paper, we present our technical contributions:

- We develop a method for observing the strictness of a function at runtime, and describe how we use generic programming to apply this mechanism to all algebraic data types (Section 3). Our technique uses only a small constant factor of overhead compared to the cost of evaluating a function without observing its strictness.

- We introduce a precise approach for specifying the strictness of functions. Such specifications are expressive enough to capture exactly how much evaluation occurs within a data structure during the evaluation of a function (Section 4). The current specification framework is relatively low-level, but crafted to allow future higher-level abstractions to build upon it, without the need to modify the core StrictCheck library.

- We work through a more complex example, Okasaki's purely functional queues [Okasaki 1995], demonstrating some of the difficulties of programming with laziness and how our approach can be used to alleviate them (Section 5).

- As a side contribution, we identify a limitation of QuickCheck's technique for generating random functions: namely, it only generates strict functions. We develop an alternate technique to generate functions with arbitrary strictness (Section 6).

- We implement StrictCheck, a Haskell framework for automatically testing functions against such specifications, available today at https://hackage.haskell.org/package/StrictCheck.

Section 7 discusses related work. We conclude and draw directions for future work in Section 8 .

\section{STRICTNESS SPECIFICATIONS, CONCRETELY}

For concreteness, suppose we want to specify the function take, which returns the first $\mathrm{n}$ elements of a given list, or the entire list if $\mathrm{n}$ is greater than its length:

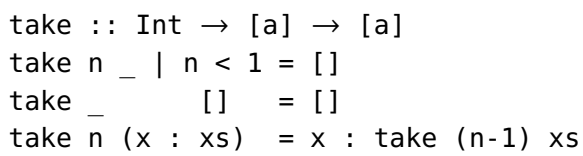

In StrictCheck, a value of the Spec type represents a strictness specification for some function. The specification of take has the type

take_spec :: Spec '[Int, [a]] [a]

This tells us that take_spec specifies a function with take's type signature, taking two arguments of types Int and [a] and returning a list of type [a].

\subsection{Writing Specs in a Curry}

In general, the Spec type can represent specifications for functions of any arity, with arguments and results of any type, including higher-order ones. Our first draft of the Spec type used heterogeneous lists to generalize over the arity of the function being specified. While this worked, the syntactic overhead of pattern-matching and constructing such lists made specifications more difficult to read. StrictCheck simplifies this syntax using arity-polymorphic currying. We briefly describe this technique before moving on with our example.

2.1.1 Arity-Polymorphic Currying. To generalize currying to any arity, we define the type family (args $\cdots \rightarrow$ result) to compute a curried function type taking args as inputs and returning result.

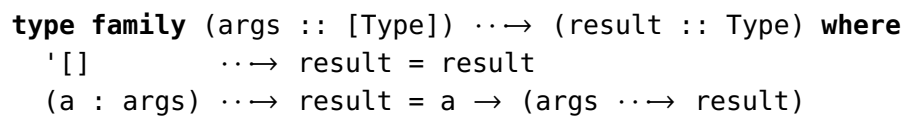


In the inverse direction, we define two type families Args and Result to compute respectively the list of arguments to a curried function, and its (non-function-type) result. For any type $f$, observe that $f \sim($ Args $f \cdots \rightarrow$ Result $f)$.

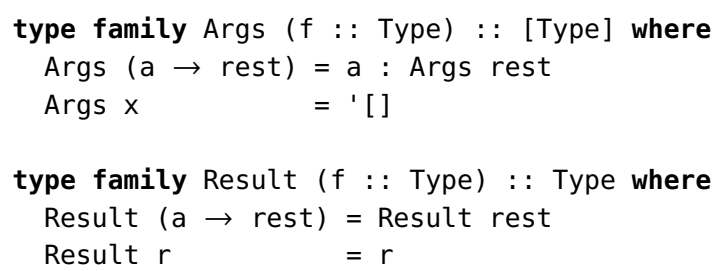

To actually curry functions, we need to pick some particular concrete type of heterogeneous lists (List : : [Type] $\rightarrow$ Type). In terms of this type, we define the Curry typeclass to curry/uncurry with our List data type:

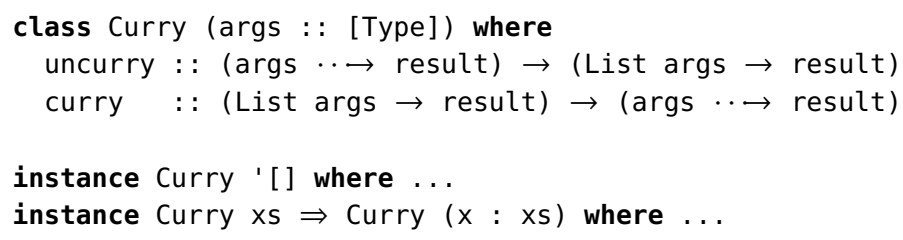

StrictCheck uses this machinery in several ways, including its specification interface.

2.1.2 Variadic Curried Specs. Because StrictCheck's Specs are defined in terms of variadic curried functions, the test author does not need to explicitly manipulate heterogeneous lists. Instead, they work within the context of a Spec, indexed by the argument and result types of the function it is meant to correspond to:

newtype Spec (args :: [Type]) (result :: Type)

$=$ Spec $($ forall $r .(\operatorname{args} \cdots \rightarrow r) \rightarrow$ result $\rightarrow(\operatorname{args} \cdots \rightarrow r)$ )

A Spec contains a specification function like those in Section 1.2, whose number of arguments is determined by the arity of the function $f$ it specifies. The wrapped specification function takes, in order:

- a curried continuation we will name predict, accepting all of f's argument types in order,

- an implicit representation of a demand on f's result (as described in Section 2.1.4), and

- all of $f$ 's original argument types in order.

The specification function will call predict on a representation of the demands which the specification author predicts $f$ will exert on its inputs, given the demand exerted on f's output. In Section 2.2, we'll see examples of such specifications in action.

2.1.3 Another Flavor of Curry. As we saw above, a Spec is indexed by two types: a list of arguments to the function specified, and the result type of that function. We might assume that a function type like ( $A \rightarrow B \rightarrow C$ ), with more than one argument, corresponds to multiple equivalent Spec types: (Spec ' $[A, B] C)$, or (Spec ' $[A](B \rightarrow C)$ ).

Perhaps surprisingly, a useful spec type should never have a function as its result type. Recall from Section 1.2 that a strictness specification maps from a demand on a function's result, as well as a list of its inputs, to a predicted demand on those inputs. Suppose we tried to specify the curried function

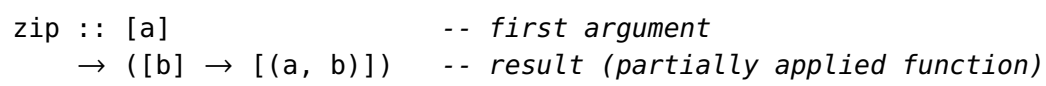


in terms of a mapping

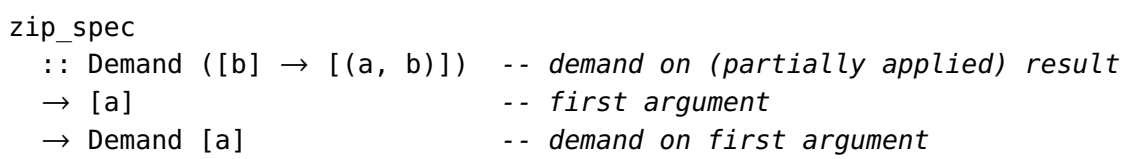

Any such specification would not be able to precisely express zip's strictness. Why? There are only two possible demands on a function: evaluated, or not evaluated. Yet the shape of the demand on zip's first argument [a] depends on how much of its result $[(a, b)]$ is evaluated by some context. A specification like this can be predicated only on whether or not the result function $[b] \rightarrow[(a, b)]$ is itself evaluated-which isn't enough information to make an exact prediction of the demand on zip's input [a].

Extending this reasoning, any exact specification of a curried function with non-trivial strictness is only expressible by uncurrying that function. In StrictCheck, types like (Spec ' $[A](B \rightarrow C)$ ) are prohibited in favor of the strictly more expressive form (Spec ' $[A, B]$ C). This conversion is handled automatically by the library; the user doesn't need to manually uncurry functions to specify them.

2.1.4 Demands as Ordinary Values? In the executable examples to follow, notice that we will overload a value of some type $\mathrm{t}$ as both an ordinary value and also as a representation of a demand on some value of that type. This might be surprising-as discussed in Section 1.1, there are more demands on values of a type than there are mere values of that type!

We will fully resolve this apparent paradox in in Section 4, but it's worth defusing some of the suspense now: when writing a specification, we embed in some value of type $t$ a representation of (Demand $t$ ) on that same type by stubbing out portions of that value with the specially "tagged" bottom value (thunk : : forall a. a). In the context of a Spec, a total value of type t corresponds to the fully strict demand on that value, while a partial value containing one or more thunks corresponds to some non-strict demand on that value.

\subsection{Let's Check Some Specs}

We're now ready to write a strictness specification for take. But what should that specification say? A good first guess might be that take will always evaluate its integer argument $n$ regardless of the demand on its result, and that it will evaluate its list argument $x$ s to the same degree as whatever demand is placed upon its result. Let's put these words into code (with type annotations for clarity):

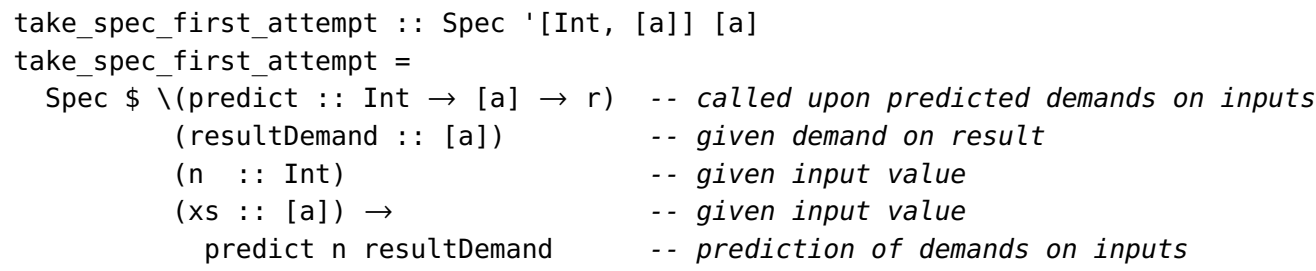

Is this specification right? Using QuickCheck [Claessen and Hughes 2000] as its backend, StrictCheck generates random inputs to the function (integers $\mathrm{n}$ and lists $\mathrm{xs}$ ), as well as random contexts in which to evaluate its result. It observes whether the evaluation induced upon the inputs to take exactly matches the prediction of the specification. Then, it shrinks any found counterexamples to a minimal form and reports them. To check our specification, we invoke StrictCheck from within GHCi: ${ }^{1}$

\footnotetext{
${ }^{1}$ Like QuickCheck, StrictCheck cannot test polymorphic functions, so we need to instantiate any type variables before testing them. Usually, the quickest way to do this is with explicit type application-as we do here to specialize take to Int.
} 


\section{ghci> strictCheckSpecExact take_spec_first_attempt (take @Int)}

StrictCheck quickly informs us that our specification does not match the actual behavior of take, printing a diagram to illustrate the mismatch (Figure 2).

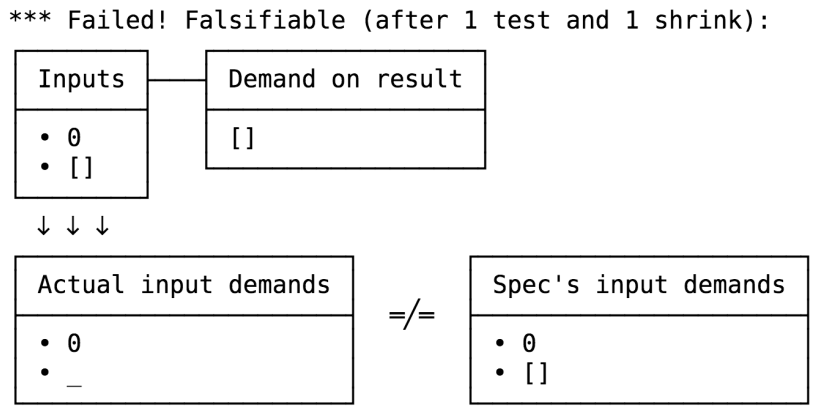

Fig. 2. Counterexample for take under the incorrect specification take_spec_first_attempt

This counterexample tells us that when we evaluated (take $\odot$ [ ] ) and fully demanded its result, [ ], our specification predicted that take would place that same demand, [ ], on the input list. However, take placed no demand on the input list, because if $n$ is zero, take immediately returns [] without evaluating the list at all.

While StrictCheck can't tell us whether a test failure indicates a bad specification or a bad implementation, in this case it is our specification is incorrect. To fix it, we need to account for what happens when take runs $\mathrm{n}$ down to zero before it gets to the end of the list. In that case, it doesn't evaluate the rest of the list, so the end of the demand on xs ought to be a thunk, not [ ]. We can correct the Spec to account for this:

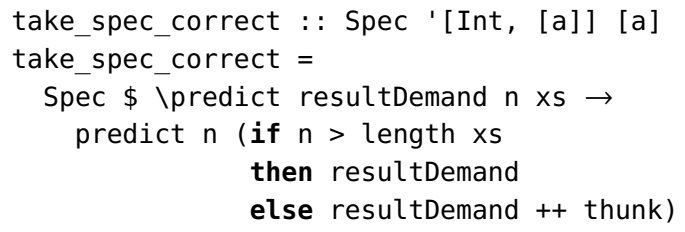

This specification passes StrictCheck's battery of random tests:

ghci> strictCheckSpecExact take_spec_correct (take @Int)

+++ 0K, passed 100 tests.

Our correct specification of take can be used to catch a variety of errors. Suppose that instead of our original definition of take, we had written the seemingly equivalent function take' :

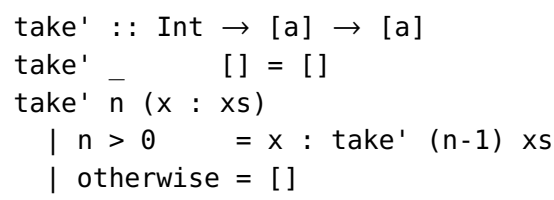

Under ordinary property-based testing, there is no way to distinguish between take and take' . They both compute the same function, but take' has different strictness than take in a fairly subtle way. Can you spot how?

Testing take' against the correct specification for take reveals that its behavior does differ from the specification (Figure 3). 


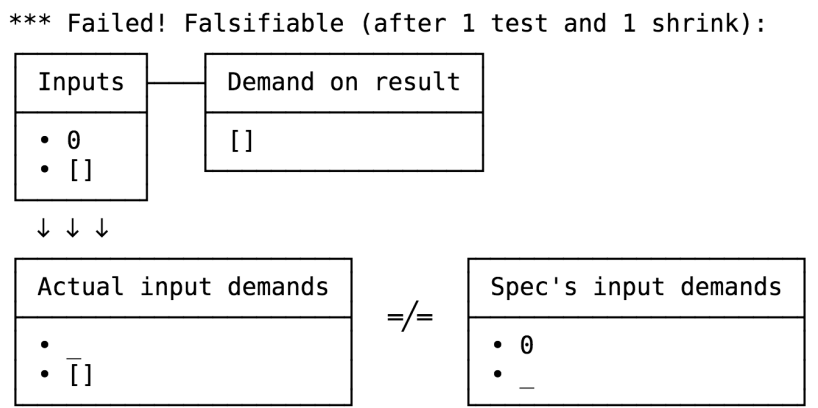

Fig. 3. Counterexample for take' under the correct specification take_spec_correct

This counterexample illustrates the strictness bug in take'. The function take evaluates $\mathrm{n}$ first, conditionally evaluating $x \mathrm{~s}$ if $\mathrm{n}$ is positive; by contrast, the function take' always evaluates the beginning of $x s$, conditionally evaluating $n$ if $x s$ is non-empty. This means that take' evaluates one more constructor of its input list than take does.

While this bug might seem trivial, it is representative of a large class of errors, many of which can be quite serious. Accidental strictness like this can lead to arbitrary extra amounts of computation. Indeed, using an overly-strict function to define a self-referencing data structure can lead to nontermination-the too-strict function can cause a thunk within the structure to depend on its own value, forming a cyclic evaluation loop.

\section{OBSERVING STRICTNESS IN HASKELL}

In order to run the above tests, StrictCheck needed to compare the predictions of a Spec with its own observation of how much input was actually evaluated by a function. In this section, we provide an efficient mechanism to record this runtime behavior.

\subsection{Getting Our Data in Shape}

In Section 1.1, we saw that a demand on a value of some type is a sub-shape-specifically, a prefix-of that value. If we were to write down the types of demands on various ordinary types, we'd find ourselves repeating a pattern. The type $D$ of demands on the values of some ordinary type $S$ :

- has the same number of constructors as $\mathrm{S}$ does,

- has the same number of fields in each constructor as $S$ does, and

- each field in D is either empty, or contains some demand on values of that field's type in S.

To actually represent such types, we define the type Thunk, to represent the possibility that some portion of a data structure has not been evaluated:

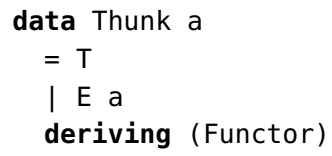

Using this type, we can say: the type of demands on values of some type is the same shape as the original type, but interleaving the Thunk type at every field, recursively throughout the entire type's shape.

Knowing this pattern, we could define the types of demands on our favorite data types, like this demand type for lists: 


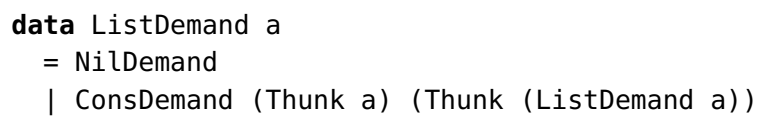

If we were to do this, though, we'd quickly be overwhelmed with the amount of boilerplate required to define such types. What's more, we'd have to write still more boilerplate to allow the demand observation mechanism to manipulate these values. We already understand the relationship between these demand types and the ordinary types to which they correspond-we ought to use this knowledge to unify these ad-hoc definitions.

To do this, we take as inspiration the concept of recursion schemes, first described by [Meijer et al. 1991] and now implemented as the modern Haskell library recursion-schemes [Kmett 2017]. This work observes that any (regular) recursive data type is isomorphic to the explicit fixed-point of some corresponding non-recursive Functor. The shape of the correct such Functor should mirror the outermost layer of the original data type, with that Functor's type parameter substituted wherever the original data type contains a recursive occurrence of itself. By factoring out the recursive structure of the original data type, the authors are able to define once and for all a suite of folds and unfolds for any data type that implements this isomorphism.

While this approach is elegant and useful, it is nevertheless not quite what we need. In particular, it is limited to expressing folds over the outermost recursive structure of the data type in question. In StrictCheck, we need to observe the evaluation of an entire data structure, into its innermost parts. The demand representation of a data type needs to interleave a Thunk at every field, not merely those which are a recursive occurrence of the original data structure.

To accomplish this, we will develop a new flavor of recursion scheme which works with structures shaped like some data type, but with a Functor (like Thunk) interleaved at every field. Our variation will let us express folds and unfolds across the entire structure of a data type, regardless of the types of its intermediate fields.

As in the original account of recursion schemes, we define a relationship between some possibly recursive type and some non-recursive type whose fixed point is shaped like the original type. We say that the Shape of a type $S$, parameterized by some Functor $f$, is the same shape as a value of $S$, but with $f$ wrapped around every field in every constructor of $S$.

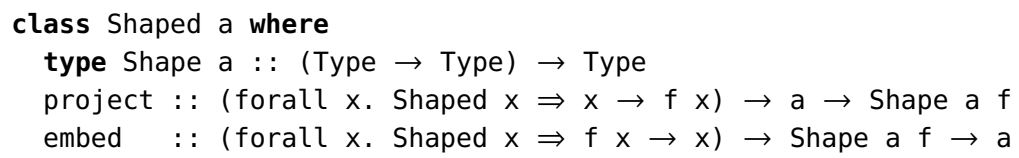

We also require every Shaped type to define projection and embedding functions to witness the relationship between the original type and its Shape. To put all this together, let's consider the data type D and a valid corresponding Shape, DShape:

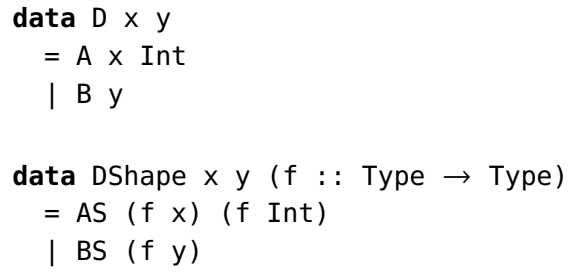

To project out the outermost layer of some $D$ out, revealing its Shape, we take some polymorphic projection function

$p:$ forall $x$. Shaped $x \Rightarrow x \rightarrow f x$ 
and call it on each field of the original value. Similarly, to embed an outer Shape back into the original type $D$, we take some polymorphic embedding function

e : : forall $x$. Shaped $x \Rightarrow f x \rightarrow x$

and call it on each field of the given Shape:

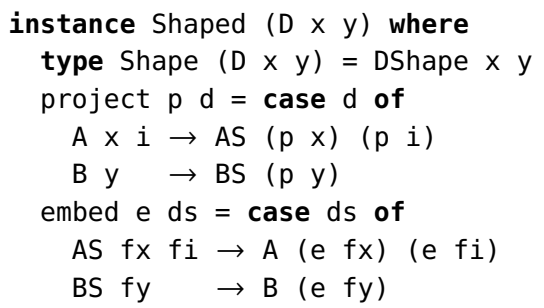

All of the above is non-recursive, dealing with only one layer of a value's structure at a time. We re-introduce recursion with an explicit fixed-point to recover the structure of our initial type. For some functor $f$, a value of type $f \% a$ is Shaped like a value of type a, but with an $f$ interleaved at every field (and around the outside of the entire value as well):

newtype ( $f::$ Type $\rightarrow$ Type) $\%$ (a : : Type) where

Wrap : : $f$ (Shape a ((\%) f)) $\rightarrow f \% a$

Unlike the isomorphism in [Meijer et al. 1991], though, there may be more (or fewer) values of this fixed-point type than of the original type, depending on the functor $f$ we interleave.

The methods of Shaped can be used to lift project and embed to operate recursively over entire interleaved structures. We call these functions interleave and fuse, respectively:

interleave : : (Functor $f$, Shaped a) $\Rightarrow($ forall $x . x \rightarrow f x) \rightarrow(a \rightarrow f \% a)$

fuse $\quad::$ (Functor $f$, Shaped $a) \Rightarrow($ forall $x . f x \rightarrow x) \rightarrow(f \% a \rightarrow a)$

These are special cases of more general derived folds and unfolds.

As we noted before, a Demand recursively interleaves Thunks into the Shape of the original data type. We represent this as the type synonym

type Demand $=(\%)$ Thunk

Using the generic programming framework Generics. SOP [de Vries and Löh 2014], we provide a default definition for the entire Shaped class which works for any Haskell 2010 algebraic data type. A user of StrictCheck who wishes to test a function on their own data type T need only ensure that it implements the Generic typeclass, then declare an empty instance of Shaped:

instance Shaped $\mathrm{T}$

An instance of Shaped for some data type is sufficient to derive observation, shrinking, and pretty-printing of demands upon its values. In the next section, we'll see how to use it for the most vital of these: observation.

\subsection{Observation, Shapefully}

Without privileged access to the runtime system, how can a Haskell program observe the evaluation of a piece of data? The "safe" subset of Haskell won't allow us to do that, so we'll need to use unsafe primitives which break referential transparency. ${ }^{2}$ In the end, we will very carefully wrap up these unsafe pieces to define a pure function observe which reports the precise demands exerted during

\footnotetext{
${ }^{2}$ We must take care to prevent compiler optimizations-many of which assume referential transparency-from breaking the functionality of these operations. For legibility, we elide these compiler hints.
} 
some function's evaluation. To illustrate this safety boundary, we'll typeset referentially opaque operations in red.

Internally, we observe evaluation by mutating reference cells during the course of a thunk's evaluation. We use the referentially opaque primitive

$$
\text { unsafePerformI0 : : I0 a } \rightarrow \text { a }
$$

to convert an ( IO a) action into a thunk which secretly runs that action and returns its result-if and when it happens to be evaluated. This operation lets us create data whose value depends on its own order of evaluation.

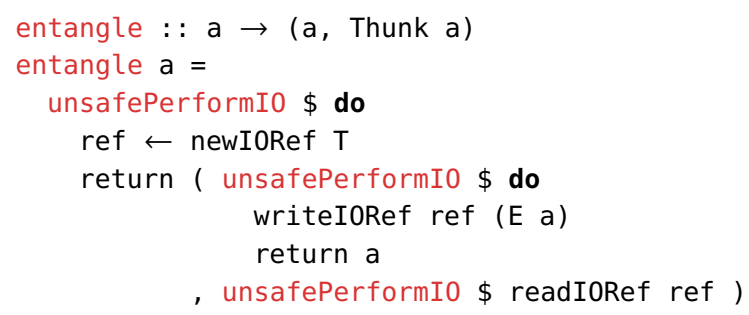

Calling entangle on some value of type A returns a pair: a copy of the original A, and a (Thunk A). The copy of the value returned by entangle is instrumented so that if and when it is evaluated, it will write its own value to a newly allocated reference cell. When the Thunk returned by entangle is evaluated, it reads from that same reference cell and returns whatever it contains at that moment. The effect of all this: if the returned A is evaluated before the corresponding Thunk, that Thunk will contain a copy of that value; if the Thunk is evaluated first, it will merely be an empty T. We can see this behavior by experimenting with entangle in GHCi:

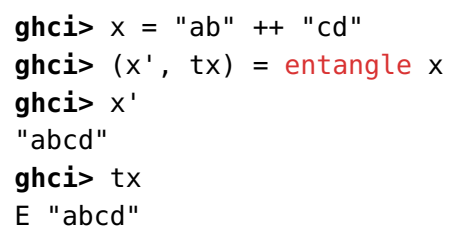

Here, because we evaluated $x$ ' first (by printing it), its value was written to the Thunk tx. If instead we evaluate the Thunk first, its value will forever be $\mathrm{T}$ :

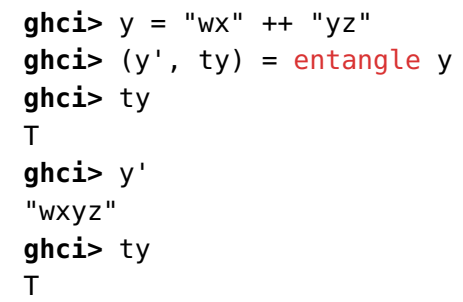

Instead of entangling just one value and a corresponding Thunk, we can use generic folds derived from Shaped to produce an instrumented copy of some value and an entire Demand reflecting the exact shape of its evaluated prefix.

To define this operation, we need to introduce one more fold, a generalized unzipWith:

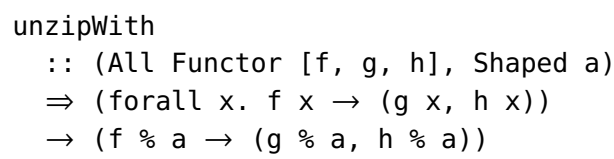


Using unzipwith as well as the fuse and interleave functions described in Section 3.1, we can recursively entangle every piece of a value.

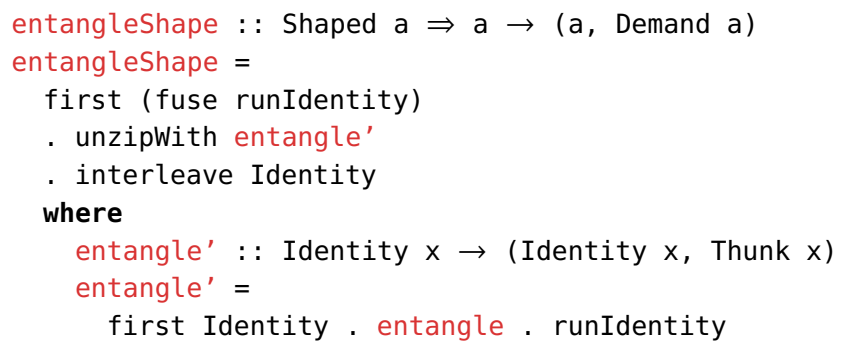

In the above, we use the Identity type

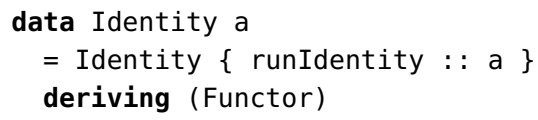

The entangleShape operation recursively unzips the structure of a value to create a mutable shadow of it. Evaluating any thunk within the resultant instrumented value triggers the update of a mutable pointer at the corresponding location in the returned Demand. When we evaluate the entangled Demand, we implicitly freeze this pointer structure, creating a reified representation of whatever sub-shape of the original value happens to be evaluated by the time the Demand itself was evaluated.

Although entangleShape is still referentially opaque, we can use it to define observe1, a pure function which observes the evaluation of a unary function on an input in a given context. We specify that context as a function context $:: b \rightarrow()$. This function will evaluate some part of the function's result before returning ( ). The caller of observel can choose what context function to provide, thus determining what demand will be placed on the function's result during observation.

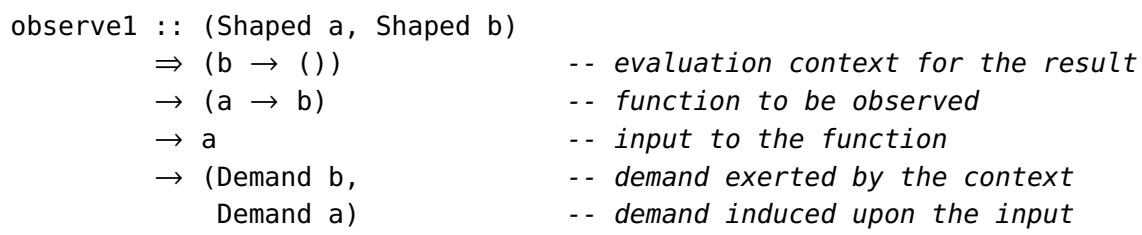

To observe the evaluation of a function on some input and in some context, observe1:

(1) entangles the input to the function, yielding an instrumented input ' and an observation of the demand upon it, inputDemand;

(2) entangles the result of the function when applied to that input, yielding an instrumented result ' and an observation of demand upon it, resultDemand;

(3) evaluates the instrumented result result' in the evaluation context; and

(4) returns the demand on the result and the demand this has induced upon the inputs.

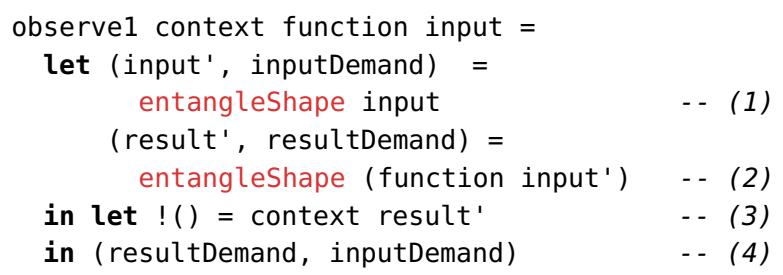


For example, observel can show us that reverse is spine-strict in its input list when its result is evaluated to weak-head normal form:

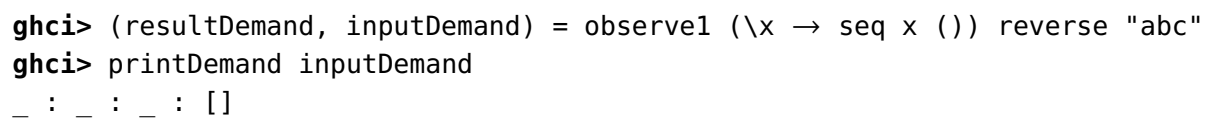

The printed demand shows us that when we demanded only the first constructor of the reversed list, every (:) and [] constructor in the spine of the list was evaluated, but none of the elements were.

To observe the evaluation of functions with more than one argument, StrictCheck provides the more general function observe which uses the variadic currying machinery in Section 2.1.

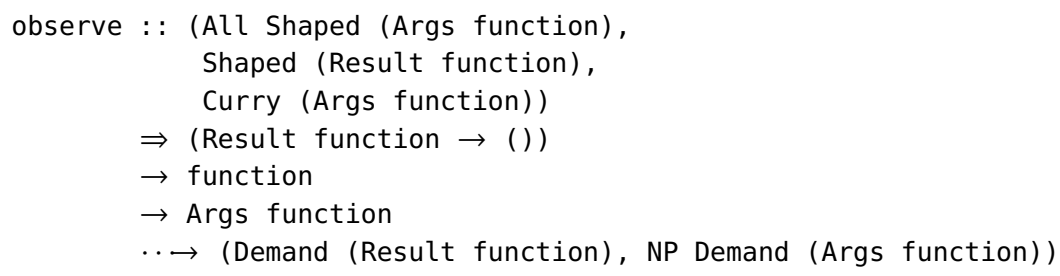

Recall that (args $\cdots \rightarrow$ result) calculates the curried function type with arguments args, returning result. The type (NP Demand (Args function)) is a heterogeneous list, each of whose elements is the induced demand observed on the function's corresponding argument. To extract these individual input demands, we pattern-match on its constructors $\left(:^{*}\right)$ and $\mathrm{Nil}$.

Using observe, let's examine the strictness of a function with multiple arguments. Consider the function productZip:

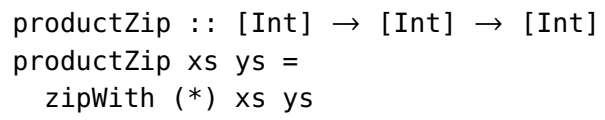

From $\mathrm{GHCi}$, we observe that in the fully strict evaluation context normalize ${ }^{3}$ the function productZip is asymmetrically strict:

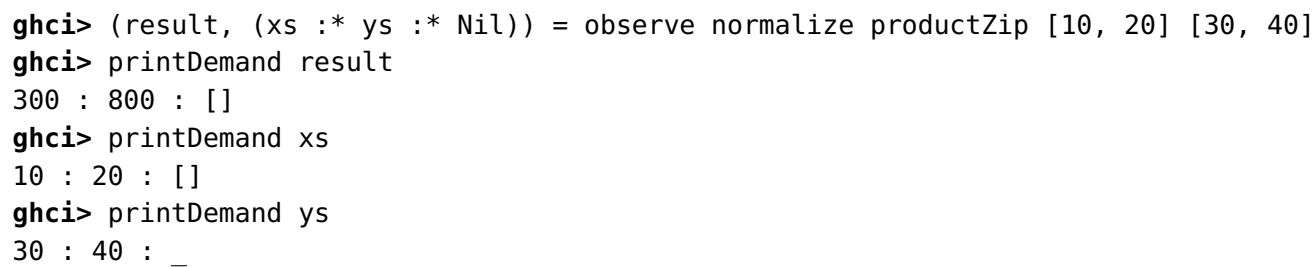

This tells us that zipwith is stricter on the left than the right: it lets the right-hand input list go untouched after it exhausts the left-hand input list.

Performance of observe. We claimed in the introduction that observing the strictness of a function has the same asymptotic performance as the original function. Previously described methods for strictness observation feed partially-undefined inputs to a function until observing that its result is not undefined [Anders Danielsson and Jansson 2004]. That method requires re-evaluating the function on many partial inputs. In the worst case, determining the function's exact strictness on some input may require evaluating it an exponential number of times (relative to the size of that input). By contrast, observe uses entangleShape to capture the exact evaluated shape of its input

\footnotetext{
${ }^{3}$ StrictCheck defines normalize as a generic operation in terms of the Consume typeclass from Section 6.2. In the context above, it suffices to think of normalize as the function foldr seq ().
} 
and result using only one invocation of the observed function. Exploring the resultant observed demand structure requires the same work to be done as would evaluating the function's ordinary result, plus a slight extra overhead to manage the mutable shadow copy of the inputs.

Purity of observe. While entangle and entangleshape are referentially opaque, observe is referentially transparent. As we saw, entangle's results are dependent on the order in which you evaluate them. On the other hand, observe takes an evaluation context as input and crucially, does not return the instrumented result of the observed function-it returns just the reified Demands. This means that any evaluation of the result of the function occurs within observe, prior to it returning any observation of demand. As such, all the updates to the mutable shape have concluded prior to our exploration of the resultant (and now frozen) demands. Because of this, the order of evaluation outside observe does not influence the value of its output.We can safely expose observe to the outside world, and fear not that it will cause untoward side effects.

\section{MANIPULATING DEMANDS IN SPECIFICATIONS}

StrictCheck specifications need to manipulate demand values in order to produce their predictions. Section 3 introduced the type (Demand : : Type $\rightarrow$ Type), which maps types to their demand types, and described how to obtain demand values by observing the evaluation of a function in some context. Now we focus on how to manipulate these values to craft precise specifications.

The writer of any kind of formal specification deserves a well-developed library for writing those specifications. If strictness specifications are to be phrased in terms of reified demands what tools will we have to manipulate these values as we build our specifications?

Take for example Haskell's standard Data. List module. Each of its many operations could be given an analogue which works over demands on lists, and each of these analogous operations might be useful to manipulate values of type Demand [a] in some specification. In principle, we could tediously implement each of these analogous operations from Data.List for Demands on lists. However, this places tremendous overhead on us, the library designers-and that's only considering specifications concerning demands on lists! We cannot reasonably expect to re-implement the rich Haskell ecosystem for our new landscape of demands.

Instead, it would be excellent if we could reuse existing functions to manipulate their corresponding demand values. However, demand values inhabit a different type. What's more, this difference in type is more than merely a difference in name: for almost all types a, there are many more inhabitants of (Demand a) than there are of a. How, then, could we automatically lift an existing function over some type a to work over the structurally distinct type (Demand a)?

\subsection{Embedding Demands in Values}

Our inspiration comes from realizing a combinatorial connection between demand types and lifted values. At every point in a Demand where a Thunk could be equal to $\mathrm{T}$, there is a corresponding point in a corresponding non-total value where some sub-part could be undefined. If we count the elements of a type (taking into account that lazy values may contain embedded bottoms), we find that there are the same number of partial inhabitants of a type as there are total inhabitants of its type of demands.

Our specifications so far have already made use of the term thunk. As alluded to in Section 2.1.4, this term is a bottom value which, when evaluated, throws a pure exception:

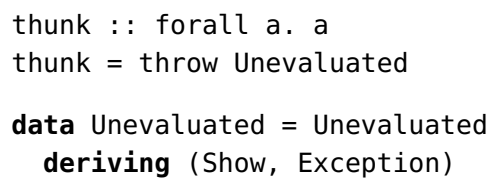


Consider the demand value $\left(\_: 2:{ }_{-}\right)$on lists of integers. Using thunk, we can represent this demand as itself a partial inhabitant of the type [Int]: (thunk : 2 : thunk). Here the first thunk represents the unevaluated Int, and the second thunk represents the unevaluated tail of the list. We can manipulate this embedded demand representation with existing list operations; for example, applying (take 1) to this list would return the singleton list [thunk] containing the first thunk.

As we might now expect, StrictCheck can losslessly translate between these new "implicit" demands and the explicit demands we've seen already. In the forward direction, it converts from a (Thunk a) to a partial value of type a by throwing the Unevaluated exception at each $T$ we encounter:

fromThunk : : Thunk a $\rightarrow$ a

fromThunk $(\mathrm{E}$ a) $=\mathrm{a}$

fromThunk $\mathrm{T}=$ thunk

In the reverse direction, it converts back from a partial value of type a to a (Thunk a) by catching the Unevaluated exception and converting it into a $\mathrm{T}$ :

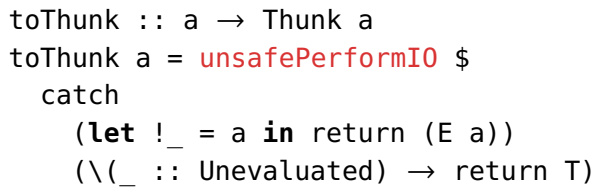

These functions are inverses, provided that every reified demand value is non-partial-that is, it contains no embedded exceptional values.

Within specifications, we may need to test whether a particular implicit demand is a thunk or not. Using toThunk, we define isThunk to detect this:

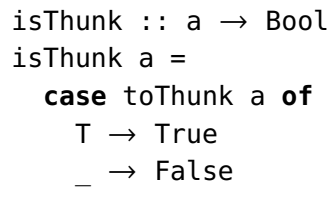

Much as entangleShape lifts entangle to operate recursively over entire demands, we can lift the above pair of inverse functions to convert entire data structures between the two representations:

toDemand : : Shaped $\mathrm{a} \Rightarrow \mathrm{a} \rightarrow$ Demand $\mathrm{a}$

Given the current Spec interface to StrictCheck, most specification authors will never need to use these conversions between demand representations. During testing, explicit demands expressed in terms of Shapes are converted to implicit demands embedded in values and back again at the boundaries of a Spec.

\subsection{Writing Specs Using Embedded Demands}

Specifications written with the Spec type are provided partial values produced by fromDemand by default. In all of the examples shown in Section 5, we manipulate demand values on lists with authentic functions from Data.List, and in these examples this approach is significantly more convenient than explicit manipulations of Demand [a] values.

When programming with these partial list values, we must be mindful of where thunk may occur. As we have seen, thunk is a divergent term. If a computation tries to evaluate thunk, its entire result becomes equal to thunk. For example, if we apply length to the partial value ( $1: 2:$ thunk), we would get back a thunk of type Int since length needs to keep evaluating the list until it reaches the [ ] constructor. Because of this, our examples will use the function (cap : : [a] $\rightarrow$ [a]), which 
replaces a thunk at the tail of a list with [ ]. This can help us prevent spine-strict functions from behaving incorrectly when lifted to implicit demands. We will see details of cap in Section 5.

We acknowledge that this design makes writing specifications more error prone than they would be if instead they explicitly manipulated Demand values and explicitly handled at every patternmatch the possibility of encountering a thunk. However, we believe this weakness to be outweighed by the expressive benefit of manipulating Demand values with any available function defined over the original data type. However, if the specification author wishes, parts of a Spec can also work with explicit Demands-the author merely needs to invoke the toDemand and fromDemand functions.

\section{CASE STUDY: PURELY FUNCTIONAL QUEUES}

In Section 2.2, we wrote a specification for the simple function take. Now, let's work through a more complex example: a specification for purely functional queues. Such queues are often implemented with two lists: a front list holding the queue elements in first-in-first-out order, and a back list holding the rest of the elements in reversed order. When the front list is empty, a dequeue operation must reverse the back list to refill the front list, which takes $O(n)$ time to finish. While an amortized analysis shows that the expected time for dequeueing is constant, this is not true if the queue is used persistently, since this $O(n)$ operation could be repeated arbitrarily many times if references to old values of the queue are re-used. In his work on purely functional data structures [Okasaki 1998], Okasaki improves upon this design using a clever application of laziness. Instead of reversing the back list all at once, Okasaki's queue incrementally performs the reversal of the back list, at the same time incrementally appending that reversed list to the end of the front list [Okasaki 1995]. Okasaki's queue relies on a function called rot:

rot $::[a] \rightarrow[a] \rightarrow[a]$

For any front and back lists, the expression ( rot front back) is functionally indistinguishable from ( front ++ reverse back). However-unlike this naïve implementation-a queue implemented with rot avoids the occasional $O(n)$ reversal, cleverly using laziness to distribute the cost of reversing the back list across each pattern-match on the front list. We'll soon see how this works in Section 5.2.

When implementing such a queue, we might accidentally break Okasaki's invariants-yet because the two implementations differ only in their strictness, we would fail to discover the mistake through traditional property testing. Fortunately, we can use StrictCheck to write a specification that lets us automatically test the strictness invariants of rot.

Before working through the tricky example of Okasaki's queue, let's first build some intuition by understanding and specifying the naïve reverse-and-append implementation of queue rotation.

\subsection{Warmup: Naïve Queues}

As earlier, there's an easy way to implement the rotate function, though this naïve version's strictness properties leave something to be desired:

rot_naive : : [a] $\rightarrow$ [a] $\rightarrow$ [a]

rot naive $f s$ bs $=f s++$ reverse bs

Before writing down a complete specification for it, it's easier to build up a picture of our predictions by considering several possible cases we could encounter while evaluating its result.

Notating Demands. Below, we'll continue to use the demand notation presented in Section 1.1. Additionally, we'll use metavariables $D_{i}$ to name either a thunk or an evaluated field. For instance, we will allow the list demand $\left(D_{1}: D_{2}: \_\right)$to denote several simultaneous possibilities, including the above 2 -cons demand (_:_:_), a similar demand where first two values are evaluated $\left(1: 2:_{-}\right)$, or a mix of both. This notation is useful to illustrate the relationship between two different demands. 
For instance, we could represent that some list demand is the reversal of another list demand by writing the first as $\left(D_{1}: D_{2}: D_{3}:[]\right)$ and the second as $\left(D_{3}: D_{2}: D_{1}:[]\right)$.

We will show the relationship of several sets of possible demands to rot_naive by arranging them into a table, using metavariables $D_{1}, D_{2}$, etc. to show how they are connected. For example, consider what happens when we make some demand $\left(D_{1}: D_{2}:_{-}\right)$upon the result of calling rot_naive on the lists $[1,2,3]$ and $[6,5,4]$ :

\begin{tabular}{c|c|c|c} 
Inputs & Output & Demand On Output & Demand On Inputs \\
\hline fs $=[1,2,3]$ & {$[1,2,3,4,5,6]$} & $D_{1}: D_{2}:_{-}$ & $\begin{array}{l}\text { fs }=D_{1}: D_{2}: \\
\text { bs }=\end{array}$ \\
bs $=[6,5,4]$ & &
\end{tabular}

In this table, the first column shows the input lists fs and bs: here, they are $[1,2,3]$ and $[6,5,4]$ respectively. The second column shows the result of (rot_naive fs bs) when fully evaluated: here, $[1,2,3,4,5,6]$. By using the metavariables $D_{1}$ and $D_{2}$, we represent several possible observations in a single table: the third column shows the demand exerted on the output, while the last column shows the demands on the inputs induced by that output demand. In this first example, where the output demand is smaller than the input list $f s$, the demand on the first argument $f s$ is equal to the demand on the output, while the demand on the second list bs is the empty demand. For example, if the output demand is $(1: 2:$ ), only the first two $(:)$ constructors of fs will be evaluated, and both will have their integer fields evaluated.

On the other hand, if the output demand is larger than the first list, then the entire spine of the second argument has to be evaluated, since determining the value of the first element of a reversed list requires reversing the entire list, all at once:

\begin{tabular}{c|c|c|c} 
Inputs & Output & Demand On Output & Demand On Inputs \\
\hline $\mathrm{fs}=[1,2,3]$ & {$[1,2,3,4,5,6]$} & $\mathrm{D}_{1}: D_{2}: D_{3}: D_{4}:$ & fs $=D_{1}: D_{2}: D_{3}:[]$ \\
bs $=[6,5,4]$ & bs $=:_{-}: D_{4}:[]$
\end{tabular}

In this case, our specification should predict that the demand on the first list will be the prefix of the output demand equal in length to fs. It should also predict that the demand on the second list will be the reverse of the remaining demand on the result, prefix-padded with thunks if necessary.

There is one last case we need to consider: when the demand on the result is equal in size to the first list, and the second list is empty:

\begin{tabular}{c|c|c|c} 
Inputs & Output & Demand On Output & Demand On Inputs \\
\hline $\mathrm{fs}=[1,2,3]$ & {$[1,2,3]$} & $\mathrm{D}_{1}: \mathrm{D}_{2}: \mathrm{D}_{3}:[]$ & $\begin{array}{l}\mathrm{fs}=\mathrm{D}_{1}: D_{2}: D_{3}:[] \\
\text { bs }=[]\end{array}$ \\
$\mathrm{bs}=[]$ & &
\end{tabular}

In this case, since the demand requires the result to be fully evaluated, we need to also evaluate the back list to ensure it is indeed empty. As such, our specification should predict that the back list will be fully evaluated, and that the demand on the front list will be identical to the demand on the result of the function.

5.1.1 Specifying Naïve Queues. Let's translate this careful case-wise analysis to write a StrictCheck specification. We use the two functions

isCapped $::[a] \rightarrow$ Bool
cap $::[a] \rightarrow[a]$

to, respectively, check if the tail of the list demand is a thunk, and replace that thunk with an empty list if so. 


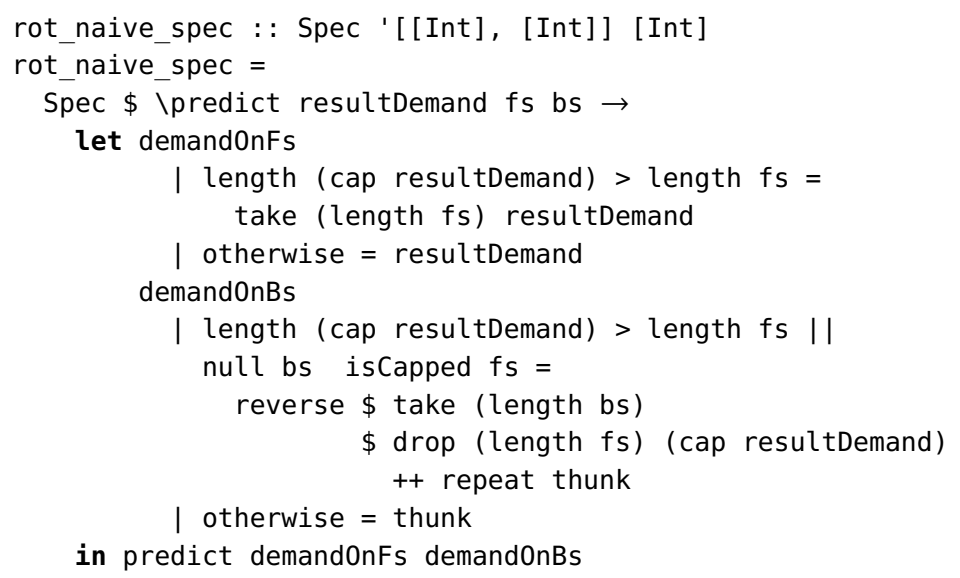

Just like the specifications of take in the introduction, this specification uses the constructor Spec applied to a function. That function has as arguments a continuation predict, the result demand resultDemand represented implicitly as a value of type [Int], (per Section 4), as well as the actual input lists fs and bs.

This specification correctly predicts the demand on fs and bs. In English, it says: "If we don't demand more from the output of rot_naive than the length of $f s$, then the demand on $f s$ is just the result demand itself, while bs is left completely unevaluated. On the other hand, if we do demand more from the output than the length of $\mathrm{fs}$, only the first piece of the demand is actually relevant to $\mathrm{fs}$, while the rest constitutes the demand on bs after being appropriately padded with thunks and reversed."

\subsection{A Trickier Spec: Okasaki’s Persistent Queues}

Now, let's specify the strictness of Okasaki's rot. The function rot is defined in terms of the more general rotate:

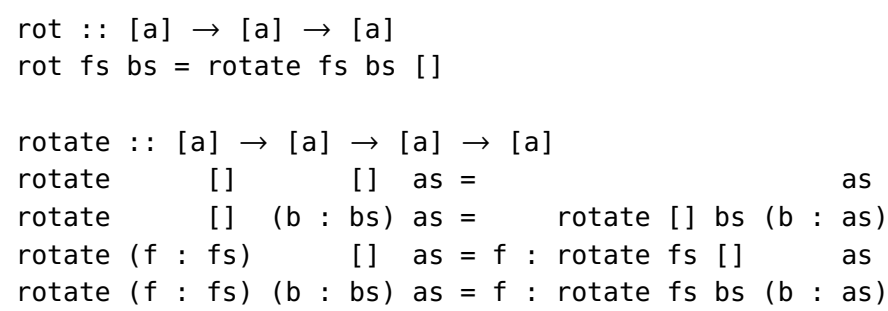

Each element taken from the result of (rot front back) requires both the front and back lists to be evaluated more deeply by one constructor. As a result, the "effort" of reversing the back list is distributed across each dequeue operation, so that by the time the front list has been notionally exhausted, the back list has already been reversed and appended to it.

It's worth noting that rotate is neither stricter nor lazier than the naïve reverse-and-append operation rot_naive. It is stricter in the back list up until the point when the front list has been exhausted-and then, it is much lazier, as there is no more work to do upon the back list.

Now, let's move on to specify the more complex rot function from Okasaki. Once again, let's build up intuition about its strictness by looking at usage examples. First, let's consider the same examples as with rot_naive. 


\begin{tabular}{c|c|c|c} 
Inputs & Output & Demand On Output & Demand On Inputs \\
\hline $\mathrm{fs}=[1,2,3]$ & {$[1,2,3,4,5,6]$} & $\mathrm{D}_{1}: \mathrm{D}_{2}:_{-}$ & $\mathrm{fs}=\mathrm{D}_{1}: \mathrm{D}_{2}:_{-}$ \\
$\mathrm{bs}=[6,5,4]$ & $\mathrm{bs}=\mathrm{I}_{-}:_{-}$
\end{tabular}

When we demand the first two elements from the result of rot, the demand propagates to a corresponding demand on the front list $\mathrm{fs}$. In fact, the demand behavior of the front list is identical to the one of rot naive. The difference between the two functions lies only in the incremental strictness when reversing the back list bs. In this example, rot_naive didn't evaluate the back list at all, since we demanded fewer elements than exist in the front list. On the other hand, rot evaluates the same number of $(:)$ cells in bs as it evaluated in $f s$. This is precisely the intuition behind Okasaki's queues: we do more work incrementally, at each step, to avoid costly $O(n)$ operations.

In our second example, the demand was larger than both lists:

\begin{tabular}{c|c|c|c} 
Inputs & Output & Demand On Output & Demand On Inputs \\
\hline $\mathrm{fs}=[1,2,3]$ & {$[1,2,3,4,5,6]$} & $\mathrm{D}_{1}: \mathrm{D}_{2}: \mathrm{D}_{3}: \mathrm{D}_{4}::_{-}$ & $\begin{array}{l}\text { fs }=\mathrm{D}_{1}: \mathrm{D}_{2}: D_{3}:[] \\
\text { bs }={ }_{-}: \mathrm{D}_{4}:[]\end{array}$
\end{tabular}

The demands in this case are identical to the ones we saw in rot_naive: they require that the spines of both lists be fully evaluated.

5.2.1 Specifying Okasaki's Queues. From this reasoning, we might write the following spec for rot by making the modifications to the specification for rot_naive which correspond to the observations above:

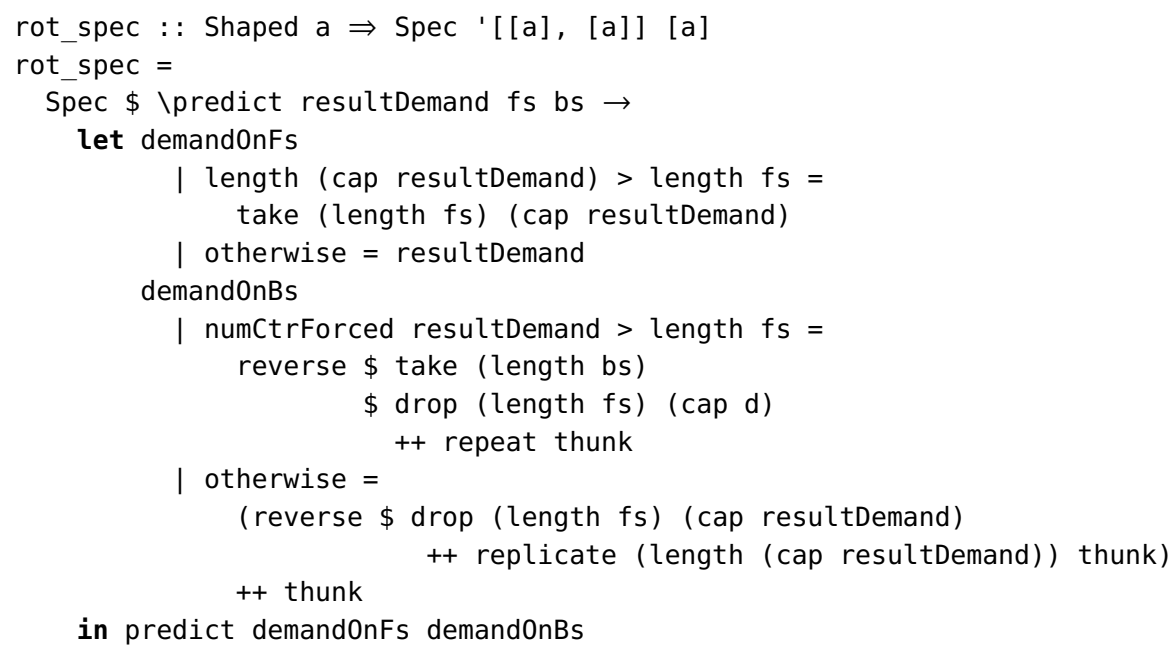

In addition to the cap helper function we saw above, this code uses another, numCtrForced, that just counts the number of evaluated constructors in the list demand, including both (: :) and [] constructors in its total. This function is implemented using the length function over lists and the previously introduced cap function that replaces the tail thunk in a list demand with an empty list.

When we test this specification using StrictCheck, it presents us with a counterexample. Repeatedly running this test reveals that all such counterexamples occur when the length of bs is greater than the length of $\mathrm{fs}$. However, this indicates a bug in our testing procedure, not in rot: Okasaki's queue implementation maintains the invariant that length $f s>=$ length $b s$, and so this case does not arise in any actual invocation of rot. However, since we didn't specify that precondition, StrictCheck identified a counterexample. The full version of the specification, including coverage 
for the case that violates the invariant, is shown in Figure 5, side-by-side with the full spec of rot_naive for easy comparison.

To conclude this section, we use StrictCheck to test the reverse and append implementation against the rot_spec specification, which immediately produces a counterexample (Figure 4).

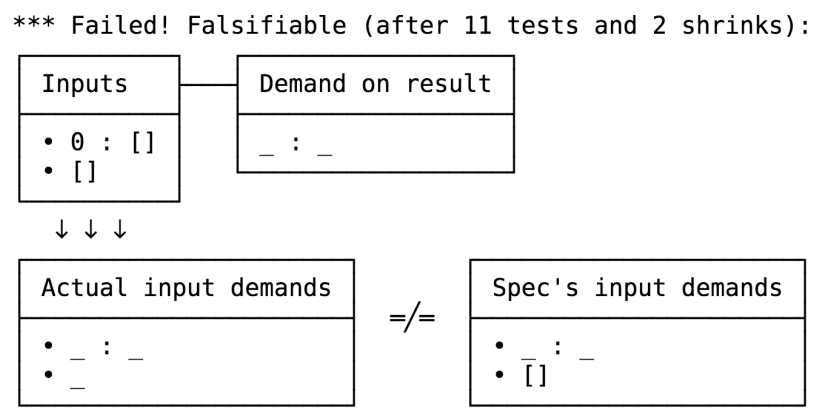

Fig. 4. Counterexample for rot_naive under rot_spec

As expected, the incremental rot specification requires the second list to be evaluated when rot_naive is in fact lazy. The specification rot_spec correctly distinguishes the incremental laziness behavior from that of rot_naive. A precise and executable specification for rot both serves both as live documentation of its intended strictness, and delivers high degree of confidence in the correctness of rot since users can easily verify its strictness behavior at the push of a button.

\section{TESTING HIGHER-ORDER FUNCTIONS}

So far, we've seen how StrictCheck can test demand specifications for first-order functions like take. Haskell is a higher-order language, though, and it would be a shame if we could only test the strictness of first-order functions. However, testing the strictness of higher-order functions comes with its own challenges. For example, consider the standard map function:

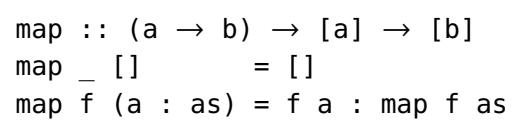

Just as with any other function, testing a strictness specification for map requires us to generate random inputs for it. In this case, we need both to generate a list of elements [a], as well as a random function $(a \rightarrow b)$. Readers familiar with QuickCheck might think to reach for its built-in CoArbitrary class, which indeed allows the user to generate random functions. As we will see, however, CoArbitrary is capable of generating only fully strict functions. This uniform strictness is fine for testing ordinary functional correctness properties, but it's a deal-breaker for StrictCheck. If we accidentally wrote a function map ' that unconditionally evaluated every element of the input list, it would behave identically to the ordinary map for all fully strict function arguments. StrictCheck would only be able to distinguish it from a correct implementation of map by testing it with a non-strict function argument. If we want to use StrictCheck to test higher-order functions, we will need to generate functions with randomly varied strictness. 


\subsection{Generating (Strict) Functions with QuickCheck's CoArbitrary}

In order to understand how StrictCheck generates random non-strict functions, it's helpful to first understand how QuickCheck generates its own random strict functions. Let's start at the beginning, by investigating its generator monad Gen.

Most users of QuickCheck only encounter Gen as an abstract type. To construct a random generator (Gen A) for a value of type A, a user combines QuickCheck's library of generator combinators (choose, elements, etc.) using Gen's monadic operations. For example, the generator below returns pairs whose first element is in the range $0-10$, and whose second element is a random boolean:

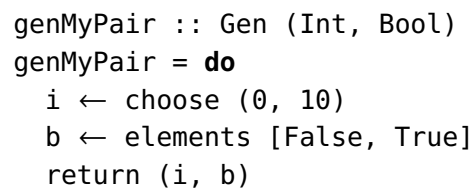

This interface lets a user describe how to build a random value without worrying about how the randomness is supplied to their generator and its component primitives. The Arbitrary typeclass defines a sensible "default" generator, meant to statistically cover every possible value in a type:

class Arbitrary a where

arbitrary :: Gen a

This approach works wonderfully for generating first-order data types. What's less clear is how to generate higher-order types like functions and data structures containing them. How might we randomly sample from a function type $(A \rightarrow B)$ ?

One easy but unsatisfying answer: if we have an Arbitrary instance for $B$, we can generate constant functions, each of which always returns some pre-generated value of $B$ :

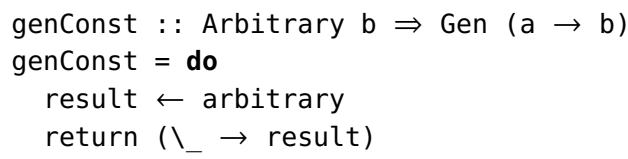

This is unsatisfying indeed: constant functions represent only a sliver of the type $(A \rightarrow B)$. There are many errors QuickCheck would never discover if it used only constant functions as higher-order inputs, as most useful tests would require us to generate functions with input-dependent results. To see how QuickCheck does this, let's peek under the hood of the Gen type to see how it really works.

In essence, a value of type (Gen A) is a function from a (pseudo-)random seed to some value of type A. When a program invokes a generator to actually create a random A, QuickCheck picks a random seed and feeds it into this function, thus returning a random A. As we've seen, the Monad instance for Gen lets users combine primitive generators to produce generators for larger data types. The monadic bind operation for generators

$(>>)$ @Gen : : Gen $a \rightarrow(a \rightarrow$ Gen $b) \rightarrow$ Gen $b$

creates a generator (Gen $b$ ) which, when given an initial random seed $r$ :

(1) splits $r$ into two statistically independent random seeds $s$ and $t$,

(2) feeds $s$ to the generator (Gen a) to produce an a, and

(3) produces $a b$ by calling the $(a \rightarrow$ Gen $b$ ) function, feeding the resultant generator with the second seed $t$.

In this way, QuickCheck ensures that different generators are not correlated.

Splitting is also the key to generating random functions with input-dependent results. To generate such functions, we need to take a generator for the function's output, and feed it with a different 
random seed depending on the value of the function's input. As a result, the function will produce different random output when given different inputs.

How might this work? For example, let's make a generator for functions (Gen (Bool $\rightarrow$ Int)). Our generator, when given an initial random seed $r$, splits $r$ into the seeds $s$ and $t$. It then picks one of $s$ or $t$, choosing based on whether the function's input Bool is True or False. Finally, it feeds that particular seed into the output generator (Gen Int). Because the choice of seed for generating the function's output is dependent on the value of the function's input, this approach generates useful non-constant functions.

Instead of asking the user to explicitly manipulate random seeds, QuickCheck provides the CoArbitrary abstraction to simplify generating random functions.

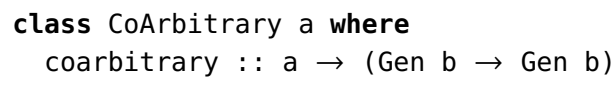

An instance of CoArbitrary for some type A describes how to use some A to perturb the random seed of some other unknown generator (Gen b) in a way that depends on the value of that $A$. To aid in writing these instances, QuickCheck exports a function called variant, which takes an integer argument and a generator, and alters the generator by perturbing its random seed in a way that depends on that integer.

variant $::$ Int $\rightarrow($ Gen $b \rightarrow$ Gen b)

To write a proper instance of CoArbitrary for A, the user should call variant at a different integer index for each different constructor of $A$, and compose the resultant perturbation function (Gen $b \rightarrow$ Gen $b$ ) with those produced recursively from the fields of that constructor. For example, here is the CoArbitrary instance for (Maybe a):

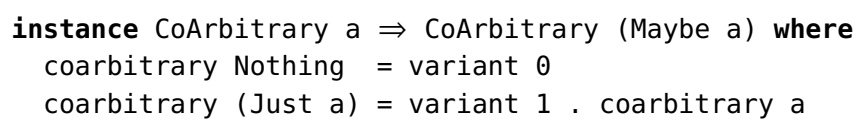

QuickCheck uses generic programming to follow this same pattern, providing a default CoArbitrary instance for most data types. Note that all correct CoArbit rary instances should destruct the entirety of their input, so that every part of an input value has a chance to perturb an output generator's random seed.

Given a CoArbitrary instance for some input type $A$ and an Arbitrary instance for some output type B, QuickCheck can generate random functions $(A \rightarrow B)$. To do this, it uses the internal function

promote : $:(a \rightarrow$ Gen $b) \rightarrow$ Gen $(a \rightarrow b)$

This operation takes a function returning generators $(a \rightarrow G e n b)$, and transforms it into a generator returning functions ( Gen $(a \rightarrow b)$ ). The generator returned by promote captures its initial random seed and returns a closure $(a \rightarrow b)$ which has access to that seed. When that closure is given an input $a$, it calls the original function ( $a \rightarrow$ Gen $b$ ) to produce a generator (Gen b). It then feeds the captured random seed to this generator to return its output $b$.

The function promote is the final piece enabling QuickCheck to generate random functions from anything CoArbitrary to anything Arbitrary:

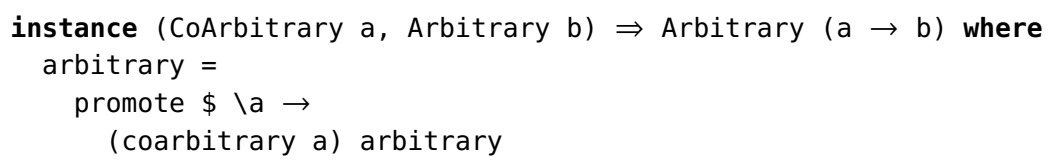

Unfortunately for StrictCheck, this approach generates only fully strict functions. Evaluating even the smallest piece of this function's result means feeding some initial random seed to its call to arbitrary. Computing this seed requires evaluating the perturbation function defined by the call 
to coarbitrary, which-if the instance of CoArbitrary is correctly defined-forces the entire input a to be fully evaluated.

Worse, even if a CoArbitrary instance didn't evaluate some of its input, we would still be unable generate functions with arbitrary strictness. Since all the random perturbation extracted from an input value is collected into a single atomic seed, evaluating that seed in turn evaluates anything that influences its value all at once. Therefore, functions generated this way exert the same demand on their input, regardless of the demand on their output.

\subsection{Generating Non-Strict Functions for StrictCheck}

To solve this problem, let's look at non-strict functions from a different perspective. We call a function productive if evaluating any finite prefix of its output requires the evaluation of only a finite prefix of its input. Consider the function zip:

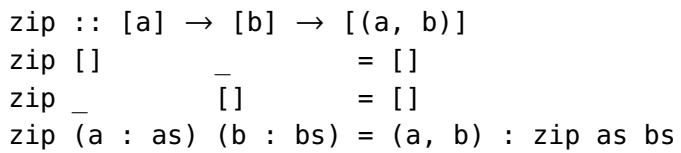

This function is productive, because evaluating a finite part of its output list requires evaluating only the corresponding pieces of its input lists. An example of a non-productive function is sum:

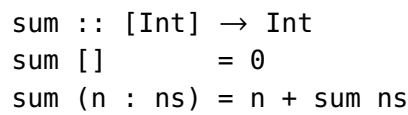

This function is not productive, because evaluating its output Int requires its entire input list to be evaluated. If its input is an infinite list, any non-trivial demand on the result of sum will cause it to diverge, as it attempts to consume all of that infinite input.

We can view a productive function as a procedure which alternates between evaluating some finite amount of input and producing some finite amount of output, until it has produced its entire output. Functions generated by StrictCheck explicitly follow this pattern, randomly alternating between consuming pieces of input and producing pieces of output. At each step, the value of some piece of input can influence both the value of some future piece of output, as well as future choices about which pieces of input to consume next.

We generate functions using two complementary typeclasses: Consume and Produce. While the CoArbitrary typeclass collapses an input all at once into a random perturbation, the Consume typeclass transforms it into a tree of individual random perturbations. As we produce pieces of input, we'll compose and apply these pieces of randomness incrementally.

We represent an individual random perturbation with the type Variant, which wraps a universal transformation on generators just like the result from QuickCheck's own variant function.

newtype Variant

= Variant $\{$ vary : : forall a. Gen a $\rightarrow$ Gen a $\}$

This type is is a Monoid: its identity and combination function are id and (. ), respectively, lifted over the newtype. We assemble individual Variants into a rose tree called an Input:

data Input

= Input Variant [Input]

The Consume typeclass, our equivalent to CoArbitrary, maps a value to an Input. The functions we'll generate will incrementally use the random perturbations it contains while producing their outputs. 


\section{class Consume a where}

consume : : a $\rightarrow$ Input

Instances of Consume create Inputs whose tree structures match those of the consumed values, with a variant at each node corresponding to the randomness derivable from the corresponding value constructors. Just as CoArbitrary provides the function variant for writing its instances, we provide the function constructor for writing instances of Consume:

constructor : : Int $\rightarrow$ [Input] $\rightarrow$ Input

constructor $i=\operatorname{Input}(\operatorname{Variant}(\operatorname{variant} i))$

Instances of Consume are defined in a similar manner to their corresponding CoArbitrary instances. For example, the Consume instance for (Maybe a) is:

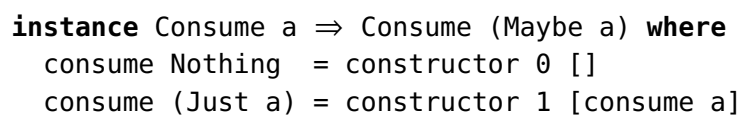

Importantly though, instances of Consume must be precisely the right strictness: evaluating the top-most Input constructor should require evaluating the top-most constructor of the consumed value, and nothing more. That is: as we evaluate an Input, the value from which it was created should be evaluated to precisely the same degree as that Input. In this way, evaluating an Input can act as a proxy for evaluating the original value to which it corresponds-a property StrictCheck relies upon for its own correctness. This rule implies that the consume method for functions must evaluate them, even though it will always return the trivial Input:

instance Consume $(\mathrm{a} \rightarrow \mathrm{b})$ where

consume ! $!_{-}$constructor $\Theta[]$

Just like CoArbitrary instances, Consume instances have exactly one correct implementation. In StrictCheck, we derive these instances automatically using generic programming.

To complement Consume, we define the Produce typeclass:

class Produce $b$ where

produce : : [Input] $\rightarrow$ Gen b

Like arbitrary, produce generates a random output value in the Gen monad. However, it also is given a list of Inputs, each of which represents a "leaf" of some still-unevaluated value. As we'll see shortly, when produce needs to recursively generate some part of its output, it destructs some of these leaves and uses the Variants they contain to further randomize its output.

For types with no fields, an instance of Produce should be equivalent to that type's Arbitrary instance. For example, the instance of Produce for Bools is merely:

instance Produce Bool where

produce $_{-}=$elements [False, True]

However, a function producing a data type with fields needs the opportunity to consume a random part of its input before it produces the value of a field. To enable this, we define a function draws, which randomly destructs some part of a list of Inputs. It collects the Variants from each Input node it traverses, returning their composition alongside the remaining leaves of the Inputs.

draws : : [Input] $\rightarrow$ Gen (Variant, [Input])

We could implement draws in many ways, each of which would give a different statistical distribution to the strictnesses of our generated functions. In StrictCheck, draws uses a geometrically bounded depth first random traversal, which biases generated functions so that demanding different pieces of output tends to evaluate different pieces of input. We detail our implementation in Appendix B. 
Using draws, we implement a function recur, which generates an output value whilst randomly consuming Inputs:

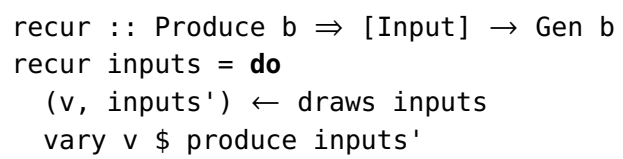

When recur is called on some list of Inputs, it invokes draws to partially consume some of those inputs. Using the random perturbation collected from the consumed Input nodes, it calls produce on the still-unconsumed leaves of Input. If produce is always mutually recursive with recur, then before a function produces a piece of output, it might randomly consume some input.

Using recur, writing a Produce instance is directly analogous to writing an Arbitrary instance. Where the user would previously have used arbitrary to produce a sub-field of a value, they substitute (recur inputs). For example, here are the Produce instances for Either $a b$, and $(a, b)$ :

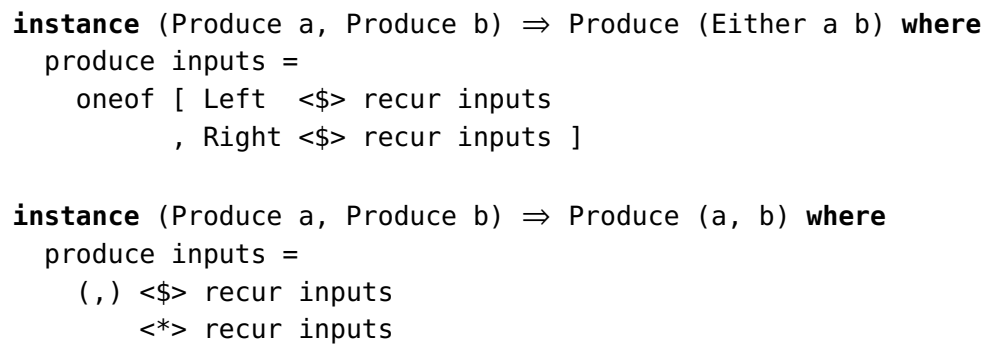

Now, let's finally use produce to generate random functions. Here, as in QuickCheck, we use promote to transform a function returning generators into a generator returning functions.

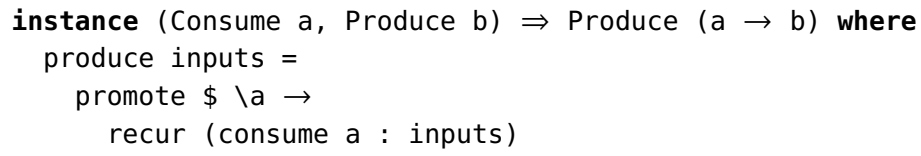

To produce a function, we consume its argument and add it to the given list of unevaluated Inputs, calling recur on those Inputs to generate its result.

We generate anything with a Produce instance in exactly this way. When testing with StrictCheck, we generate all arguments (including first-order ones) using the polymorphic generator nonStrict:

nonstrict : : Produce $a \Rightarrow$ Gen a

nonstrict $=$ produce []

This generator is equivalent to arbitrary for first-order arguments, but as we've seen, generates higher-order arguments of arbitrary strictness.

\subsection{Specifying Higher-Order Functions}

Now that we can properly generate random non-strict functions, let's test the strictness properties of higher-order functions. For example, let's specify and test the strictness of map:

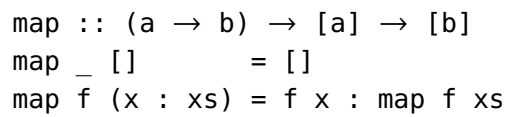

In order to specify map, we need to introduce a new specification combinator, specifyl, which derives a trivially correct specification for a unary function: 


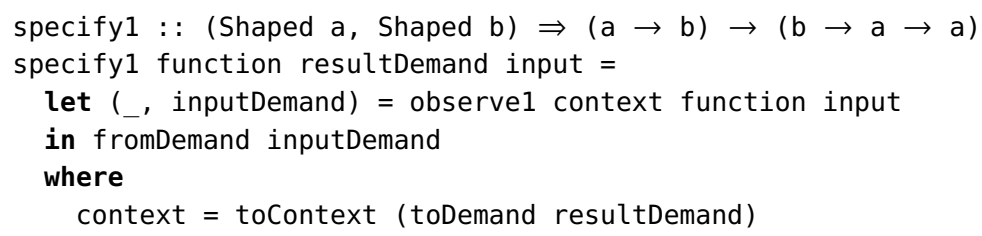

This function takes a function, an implicitly-represented demand on its result, and its input, using observe1 to see what it actually does under the context corresponding to that demand. It uses the function (derived from Shaped)

toContext : : Shaped $\mathrm{b} \Rightarrow$ Demand $\mathrm{b} \rightarrow(\mathrm{b} \rightarrow())$

to convert a demand into an evaluation context suitable for observation.

We'll use specifyl to reify the strictness behavior of a generated higher-order argument, so our specification can depend on it. Consider the difference in strictness between the functions (map (const 1)) and (map id)-a correct specification of map needs to depend on the strictness of map's argument, as this will determine how strict map is as a whole on its input list. With specifyl in hand, we can correctly specify map:

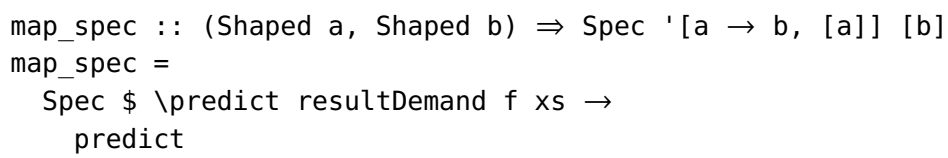

This specification predicts that the function given to map will be evaluated only if the demand on map's result evaluates at least one element of the list. Additionally, each element of the input list is evaluated to precisely the degree required by map's function argument, under the particular demand placed on the corresponding element of the result list. This specification passes all tests.

\section{RELATED WORK}

StrictCheck is the first framework in Haskell for property-based testing of strictness with exact specifications. There is a rich body of work on property-based testing, observing lazy programs, and working with partial values in Haskell. We discuss their relationship to StrictCheck, and comment on StrictCheck's novelty compared to existing work.

Property-based testing. QuickCheck [Claessen and Hughes 2000] focuses on testing functional properties of Haskell programs through user-provided property specifications. StrictCheck uses QuickCheck's type-based random generator as its backend for generating random inputs, but focuses on testing strictness (traditionally considered a non-functional property) against user-provided specifications. StrictCheck also provides a more flexible variant of the CoArbitrary typeclass from QuickCheck, capable of generating functions with random strictness.

SmallCheck and Lazy SmallCheck [Runciman et al. 2008] are similar to QuickCheck, and provide property-based testing of functional properties against a specification. They differ from QuickCheck by exhaustively checking properties on inputs up to a certain depth instead of, as QuickCheck does, randomly sampling from a large space of values. Lazy SmallCheck allows property-based testing by generating partial values as inputs, but there the purpose is to verify functional properties on partial inputs. Lazy SmallCheck does not provide exact strictness specification in the style of StrictCheck. 
Observing Haskell programs. In [Gill 2001], Gill develops an (unnamed) library for observing the evaluation of Haskell values. Gill's work uses a similar technique of injecting effectful code into values through unsafePerformI0, but his work only records the evaluated values as strings, while StrictCheck uses a typed approach that fully reifies the evaluated structure as a first class value which may be manipulated by other Haskell programs. Gill's library provides programmers with runtime information to aid in manual debugging of lazy functional programs, whereas StrictCheck provides automated testing of strictness on such programs.

Haskell libraries such as ghc-heap -view [Breitner 2014] and Vacuum [Morrow and Seipp 2009] provide functions for inspecting the heap representation of Haskell values at runtime. These libraries can reify pointer graphs describing the current heap state of the inspected value. StrictCheck's observation mechanism is different from these libraries: we observe the strictness of a function rather than the evaluation structure of a data value. StrictCheck's observe mechanism is referentially transparent, whereas these libraries operate in the I0 monad.

Programming with partial values. Danielsson et al. developed the ChasingBottoms library in Haskell in order to study program verification under the context of partial and infinite values [Anders Danielsson and Jansson 2004]. The ChasingBottoms library provides a set of functions to test whether a Haskell value is divergent. StrictCheck uses similar techniques to convert reified demand values to and from partial values.

Testing strictness. Chitil published a framework for testing the strictness of Haskell functions also named StrictCheck [Chitil 2011]. Chitil's work develops a notion of "least-strictness", and tests whether a function is least-strict by feeding partial values as inputs to the function. This only tests a very specific strictness property of Haskell functions, while our work allows users to precisely specify and test a broader category of strictness property. StrictCheck also generalizes to higher-order functions, a domain not addressed in this prior work.

\section{CONCLUSION AND FUTURE WORK}

In this paper, we identified a class of dynamic properties typically considered out of scope for traditional property-based testing: strictness properties. We described an approach to specify, observe, and automatically test such properties and implemented it in an openly available Haskell library called StrictCheck.

Since this approach is somewhat new, there is a lot of space for improvement. In particular, the specification language is relatively low-level. A more declarative and general specification language could be constructed atop StrictCheck's foundations, and we anticipate exploring this design space in future work. Even without a high-level specification language, StrictCheck can be used to test whether a function has the same strictness as a reference implementation. Additionally, the same technique could be used to identify buggy compiler optimizations that break strictness. Further, taking inspiration from [Claessen et al. 2010], we would like to synthesize strictness specifications for functions based upon dynamic observations of their behavior. 


\section{A COMPARING THE SIMPLE AND FULL SPECIFICATIONS OF QUEUE ROTATION}

rot_simple_spec :: Spec '[[Int], [Int]] [Int]

rot_simple_spec $=$

Spec $\$ \backslash$ predict resultDemand fs bs $\rightarrow$

let demand0nFs

| length (cap resultDemand) > length fs = take (length $\mathrm{fs}$ ) resultDemand

| otherwise $=$ resultDemand demand0nBs

| length (cap resultDemand) > length fs

II null bs iscapped $\mathrm{fs}=$

reverse \$ take (length bs)

\$ drop (length fs) (cap resultDemand)

++ repeat thunk

| otherwise $=$ thunk

in predict demand0nFs demand0nBs rot_spec :: Spec '[[Int], [Int]] [Int]

rot_spec =

Spec $\$ \backslash$ predict resultDemand fs bs $\rightarrow$

let demand0nFs

| length (cap resultDemand) > length fs = take (length fs) (cap resultDemand)

| otherwise $=$ resultDemand

demand0nBs

I numCtrForced resultDemand $>$ length fs $=$ - Identical to rot simple case

reverse $\$$ take (length bs)

\$ drop (length fs) (cap resultDemand) ++ repeat thunk

-- Only needed when length bs > length fs

| length (cap resultDemand) > length bs = reverse \$ drop (length fs) (cap resultDemand)

++ replicate (length bs) thunk

- Force part of bs even if not demanded

otherwise $=$

(reverse \$ drop (length fs) (cap resultDemand)

++ replicate (length (cap resultDemand)) thunk) ++ thunk

in predict demand0nFs demandOnBs

Fig. 5. StrictCheck specifications of rot simple and rot side by side, as described in Section 5.2 


\section{B IN DETAIL: INTERLEAVING RANDOM EVALUATION INTO GENERATION}

The function draws described in Section 6.2 evaluates a random sub-forest of some [Input] in a random depth-first order. In the end, it returns a Variant storing the combined entropy from all the nodes of the Inputs it traversed, as well as a new forest of the yet-to-be-consumed "leaves" of the original Inputs. The number of nodes of Input consumed by a call to draws is given by a geometric distribution with expectation 1 . Below we list the full implementation of draws, presented alongside a selection of the Produce and Consume APIs for reference.

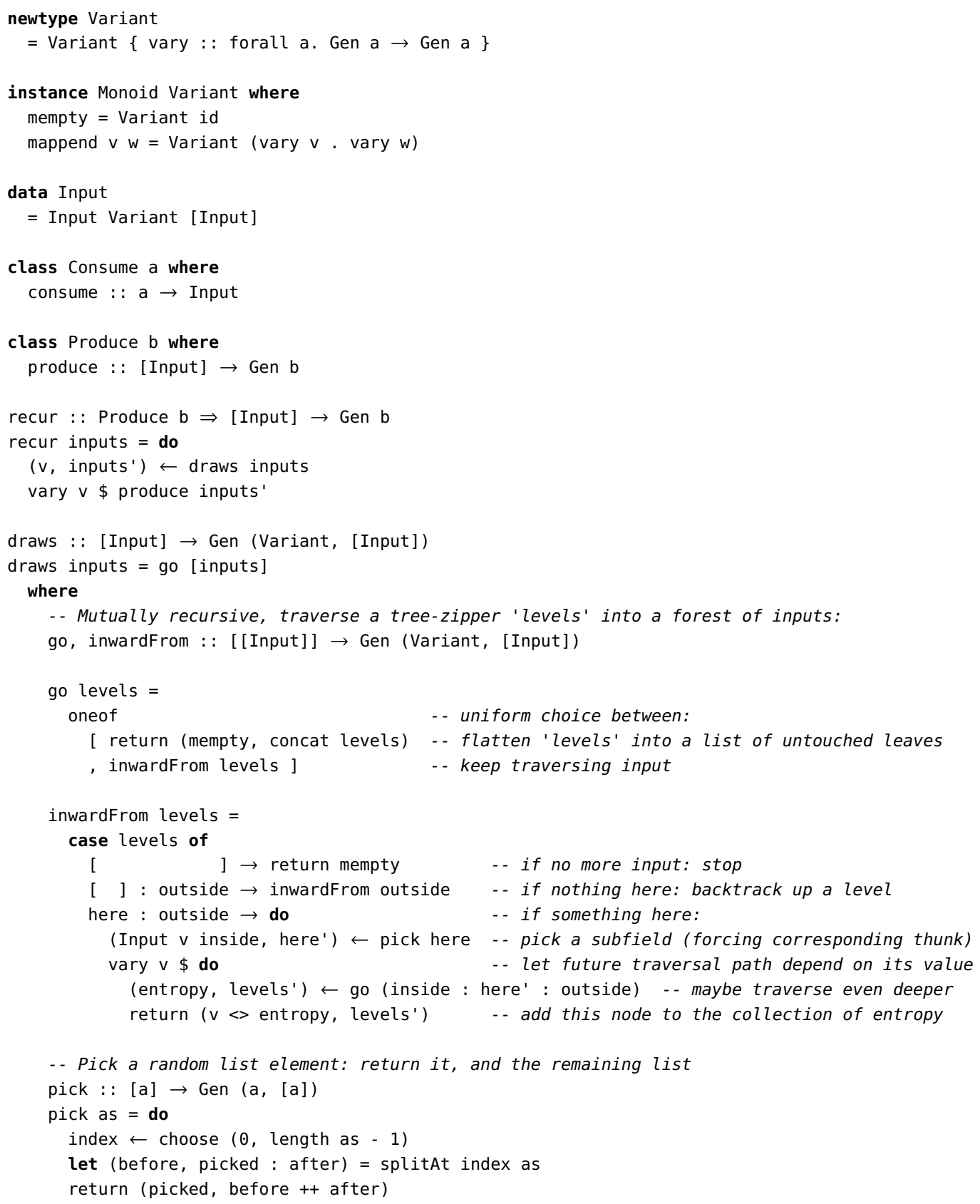




\section{ACKNOWLEDGMENTS}

We are grateful to José Manuel Calderón Trilla, Stephanie Weirich, Benjamin Pierce, Mayur Naik, Katrina Xiaoyue Yin, Jennifer Paykin, Robert Rand, Antal Spector-Zabusky, Matthew Weaver, Ryan Trinkle, Daniel Winograd-Cort, Sandra Dylus, Juliette Martin, and the Penn PLClub for their useful comments and support. One of the authors developed an early version of this work in a streamed programming session; the authors would like to thank those who participated. This material is based upon work supported by the National Science Foundation under Grant Numbers 1421243, 1521523, 1703835, and 1319880. Any opinions, findings, and conclusions or recommendations expressed in this material are those of the authors and do not necessarily reflect the views of the National Science Foundation.

\section{REFERENCES}

Andreas Abel, Brigitte Pientka, David Thibodeau, and Anton Setzer. 2013. Copatterns: Programming Infinite Structures by Observations. In Proceedings of the 40th Annual ACM SIGPLAN-SIGACT Symposium on Principles of Programming Languages (POPL '13). ACM, New York, NY, USA, 27-38. https://doi.org/10.1145/2429069.2429075

Nils Anders Danielsson and Patrik Jansson. 2004. Chasing Bottoms - a Case Study in Program Verification in the Presence of Partial and Infinite Values. (05 2004).

Joachim Breitner. 2014. ghc-heap-view: Extract the heap representation of Haskell values and thunks. https://hackage haskell.org/package/ghc-heap-view. (2014). [Online; accessed 14-March-2018].

Olaf Chitil. 2011. StrictCheck: a Tool for Testing Whether a Function is Unnecessarily Strict. Technical report 2-11. University of Kent, Kent, UK. 182-196 pages. http://kar.kent.ac.uk/30756/

Koen Claessen and John Hughes. 2000. QuickCheck: A Lightweight Tool for Random Testing of Haskell Programs. SIGPLAN Not. 35, 9 (Sept. 2000), 268-279. https://doi.org/10.1145/357766.351266

Koen Claessen, Nicholas Smallbone, and John Hughes. 2010. QuickSpec: Guessing Formal Specifications Using Testing. In Tests and Proofs, Gordon Fraser and Angelo Gargantini (Eds.). Springer Berlin Heidelberg, Berlin, Heidelberg, 6-21.

Edsko de Vries and Andres Löh. 2014. True sums of products. In WGP@ICFP.

Andy Gill. 2001. Debugging Haskell by Observing Intermediate Data Structures. Electronic Notes in Theoretical Computer Science 41, 1 (2001), 1. https://doi.org/10.1016/S1571-0661(05)80538-9 2000 ACM SIGPLAN Haskell Workshop (Satellite Event of PLI 2000).

Ralf Hinze. 2000. Memo Functions, Polytypically!. In Proceedings of the 2nd Workshop on Generic Programming, Ponte de. 17-32.

J. Hughes. 1989. Why Functional Programming Matters. Comput. f. 32, 2 (1989), 98-107. https://doi.org/10.1093/comjnl/32.2.98

Edward A. Kmett. 2017. recursion-schemes: Generalized bananas, lenses and barbed wire. http://hackage.haskell.org/ package/recursion-schemes. (2017). [Online; accessed 12-June-2018].

Erik Meijer, Maarten Fokkinga, and Ross Paterson. 1991. Functional Programming with Bananas, Lenses, Envelopes and Barbed Wire. Springer-Verlag, 124-144.

Matt Morrow and Austin Seipp. 2009. vacuum: Graph representation of the GHC heap. https://hackage.haskell.org/package/ vacuum. (2009). [Online; accessed 16-March-2018].

Chris Okasaki. 1995. Simple and efficient purely functional queues and deques. JOURNAL OF FUNCTIONAL PROGRAMMING 5, 4 (1995), 583-592.

Chris Okasaki. 1998. Purely Functional Data Structures. Cambridge University Press, New York, NY, USA.

Colin Runciman, Matthew Naylor, and Fredrik Lindblad. 2008. Smallcheck and Lazy Smallcheck: Automatic Exhaustive Testing for Small Values. In Proceedings of the First ACM SIGPLAN Symposium on Haskell (Haskell '08). ACM, New York, NY, USA, 37-48. https://doi.org/10.1145/1411286.1411292 